\title{
Bernadette DUARTE (dir.), Les pratiques religieuses alimentaires : limiter le risque discriminatoire
}

Paris, L'Harmattan, 2016

\section{Candice Bordes}

\section{CpenEdition}

\section{Journals}

Édition électronique

URL : https://journals.openedition.org/rdr/609

DOI : $10.4000 /$ rdr.609

ISSN : 2534-7462

Éditeur

Presses universitaires de Strasbourg

Édition imprimée

Date de publication : 31 octobre 2017

Pagination : 215-217

ISBN : 978-2-86820-974-0

ISSN : 2493-8637

\section{Référence électronique}

Candice Bordes, «Bernadette DUARTE (dir.), Les pratiques religieuses alimentaires : limiter le risque discriminatoire », Revue du droit des religions [En ligne], 4 | 2017, mis en ligne le 15 janvier 2020, consulté le 23 mai 2022. URL : http://journals.openedition.org/rdr/609 ; DOI : https://doi.org/10.4000/ rdr.609

Ce document a été généré automatiquement le 23 mai 2022

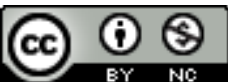

La revue du droit des religions est mise à disposition selon les termes de la Creative Commons Attribution - Pas d'Utilisation Commerciale 4.0 International - CC BY-NC 4.0. 


\title{
Bernadette DUARTE (dir.), Les pratiques religieuses alimentaires: limiter le risque discriminatoire
}

Paris, L'Harmattan, 2016

\author{
Candice Bordes
}

\section{RÉFÉRENCE}

Bernadette DUARTE (dir.), Les pratiques religieuses alimentaires : limiter le risque

discriminatoire, Paris, L'Harmattan, 2016 (Droit, Société et Risques), 300 p.

1 Cet ouvrage collectif s'interroge sur les pratiques religieuses alimentaires, réflexion plutôt récente et également délicate, particulièrement en France. Tout l'intérêt de cette analyse est de soulever les questions relatives à ces "codes comportementaux » ou «marqueurs d'identité religieuse» (Nadine Weibel, p. 33) en théorie comme en pratique, et en développant notamment une approche comparée avec la Belgique et le Canada. Il est immédiatement rappelé que ces pratiques s'inscrivent dans un cadre plus large incluant le principe de laïcité, la liberté de conscience et la liberté de manifestation des croyances qui en découle. Ces derniers éléments sont communément connus à travers différents textes. Ces dispositions restent cependant très générales même s'il peut être considéré que «la liberté de religion comprend la liberté de manifester ses croyances, y compris par l'observation de prescriptions alimentaires » (Bernadette Duarte, p. 17).

2 Malgré tout, des difficultés peuvent apparaître. La première suppose d'éclairer les esprits sur la réalité de ces pratiques, et ceci dans le cadre de différentes religions: judaïsme, christianisme, islam, hindouisme ou encore bouddhisme. Par cet intermédiaire, l'accent est mis sur la diversité des pratiques religieuses alimentaires et la diversité de leur interprétation, cela également au sein d'une même religion. «La nourriture est porteuse de sens est peut être mise en lien avec le sacré » 
(Nadine Weibel, p. 34). Ainsi ces pratiques peuvent être vectrices de rassemblement comme d'éloignement, selon ce qui est considéré comme pur ou impur dans la consommation comme dans le processus de préparation des aliments.

3 Une seconde difficulté vient du fait que les pratiques alimentaires peuvent être abordées sous des aspects bien différents de la religion; elles ont également des implications culturelles, économiques ou encore politiques, et «ce constat explique peut-être l'absence d'un cadre juridique précis et spécifique à cette question» (p. 83) : c'est un droit «en construction» (Pierre-Henri Prélot, p. 87). De nombreux intervenants considèrent à ce titre que le cadre juridique français est défaillant en la matière. Ces lacunes imposent de s'adapter en pratique au cas par cas, il faut alors mettre les intérêts en balance dans le but d'atteindre un certain équilibre. Concernant les établissements pénitentiaires par exemple, il est toujours question d'une « recherche quotidienne et incessante d'un équilibre entre les individus et les groupes, entre les personnes détenues et les personnels, entre les intérêts personnels et les intérêts collectifs et entre les intérêts individuels et l'intérêt général » (Marion Zatti, p. 187). Mais d'autres acteurs sont concernés par ces questions, tant dans le secteur public que le secteur privé. En cantine scolaire ou dans le milieu militaire (Capitaine Julien Jeanmougin, p. 179) se pose par exemple la question de la compatibilité des pratiques religieuses alimentaires avec la qualité nutritive des repas qui doivent être fournis en fonction des activités à réaliser. Dans le milieu hospitalier, il s'agit de ne pas mettre en danger la santé du patient (Carine Kot, p. 203). Concernant les entreprises, pour les employés par exemple, les pratiques telles que le ramadan peuvent amener à repenser l'organisation globale des horaires de travail (Laurence Fornari, p. 212). Mais ce qui transparaît clairement dans tous les domaines d'activité évoqués, est que «concrètement, c'est le pragmatisme qui prévaut» pour appréhender ces problématiques (Eric Thorel, p. 168).

4 La conciliation est la solution retenue par de nombreuses institutions, notamment en Belgique (Patrick Charlier, p. 111). Ces solutions s'inscrivent alors dans le sillage plus large du principe de non-discrimination et prévoient souvent la mise à disposition de menus alternatifs, par exemple des menus "sans porc ", "sans viande " ou encore " végétariens ». L'expérience canadienne donne également un exemple d'adaptation en la matière par l'intermédiaire des «accommodements raisonnables" obligatoires. Ainsi, selon la requête qui est faite, un accommodement sera accordé s'il ne suppose pas une contrainte excessive pour le service qui doit le mettre en place. L'accommodement doit garantir le respect de la dignité humaine, de la liberté de conscience, de la liberté de religion mais aussi de l'égalité. Une contrainte excessive supposerait quant à elle de remettre en cause par cet accommodement les droits d'autrui, les conditions de sécurité, la conduite des opérations menées par le service ou entraînerait un coût financier trop important. Ainsi, « les pratiques d'accommodement alimentaire qui sont observées au Québec, de même que dans l'ensemble du Canada, sont empiriquement élaborées et localement mises en œuvre, tout en étant fondées en droit » (Pierre Bosser, p. 147). En France, ces accommodements ont été évoqués par le rapport Stasi du 11 décembre 2003 qui estime que «l'esprit de la laïcité requiert cet équilibre des droits et des devoirs ». S'il est bien fait mention des accommodements raisonnables, un doute peut toutefois porter sur l'interprétation qui en est faite, car elle ne semble pas aussi proche de la conception canadienne qu'il y paraît. 
5 Finalement, les contributions et témoignages de cet ouvrage collectif mettent en relief un trait caractéristique concernant l'appréhension des pratiques religieuses alimentaires : les solutions adoptées restent des réponses de circonstances. Dans ce sens, face à "l'émergence d'une religiosité à la carte » induite par un phénomène d'individualisation des pratiques religieuses (François Mabille, p. 63), et sans la mise en place d'un cadre juridique spécifique et déterminé, c'est une « laïcité à la carte » qui se développe également (Sébastien Leprêtre, p.161). Malgré tout, il est possible de remettre en perspective l'ensemble de ces recommandations et revendications à la lumière d'autres analyses. Ces théories estiment au contraire que l'intervention du législateur en la matière ne serait pas forcément pertinente et ne serait pas même souhaitable. En effet, cette intervention pourrait justement être paralysante pour les entités confrontées à ces questions en pratique. "Exiger une trop grande rigidité pourrait se révéler néfaste lorsqu'il est souhaitable de laisser une marge de manœuvre aux autorités nationales pour apprécier certaines situations. » Encore une fois, la recherche d'un équilibre est primordiale et «la neutralité de l'État ne doit pas se traduire par une société aseptisée » (Bernadette Duarte, p. 250).

\section{AUTEURS}

\section{CANDICE BORDES}

Allocataire de recherche en droit public, Université de Perpignan Via Domitia, Centre du droit économique et du développement (CDED) 